\title{
Overview of Risk factors of diabetic macular edema treatment prognosis
}

\author{
Abdulrhman Ali Hassan Hassan, Ahmed Abdullah Yahya Ojaem, Mohammed Nasser Ahmed \\ Asiri, Sarah Hassan Asiri
}

Faculty of medicine, King Khalid University, Abha, Saudi Arabia

\begin{abstract}
:
Background: Diabetes mellitus (DM) is just one of the world's fastest growing chronic conditions as well as a leading cause of acquired vision loss, diabetic macular edema (DME) is a disease linked to diabetes. Some individuals with diabetes will certainly get a kind of retinopathy-a problem of the retina in the eye. Diabetic retinopathy could progress as well as result in DME (additionally referred to as retinal swelling) that might impact your vision, and is among the most usual reasons for blindness.

Objective: In this review we discuss about the most dangerous risk factors during treatment and in daily life, which can worsen the treatment prognosis.

Methods: PubMed and Embase database were searched up to July, 2018 for relevant studies that discussing the risk factors of glaucoma.

Result: It is clear that the variety of people affected by DM is expanding greatly, as is the variety of patients with problems from DM. Fortunately; the eye symptoms of DME can be identified and managed prior to considerable vision loss happens if patients with DM undergo routine eye tests and much better manage their systemic condition.

Conclusion: Close collaboration in between doctors and also ophthalmologists is important in the managing of patients with diabetic retinopathy and also DME. To make certain very early medical diagnosis as well as treatment all diabetic person patients should be screened for diabetic person retinopathy. Control of blood pressure, serum lipids, and also renal function are very important treatments focused on prevention of visual impairment associated with diabetic retinopathy and DME. Keywords: Risk factors, diabetic macular edema, treatment, prognosis.
\end{abstract}

\section{Introduction:}

Diabetes mellitus (DM) is just one of the world's fastest growing chronic conditions as well as a leading cause of acquired vision loss [1]. According to the World Health Organization, it is estimated that the total variety of people with diabetes will double from 171 million in 2000 to 366 million by $2030{ }^{[2]}$. Diabetic retinopathy (DR), a details microvascular difficulty of DM, continues to be the leading root cause of obtained vision loss worldwide in middle-aged as well as therefore economically active individuals ${ }^{[1]}$.With the enhancing number of individuals with diabetes mellitus, the variety of DR and vision-threatening DR (VTDR), that includes serious non-proliferative DR, proliferative DR (PDR) as well as diabetic person macular edema (DME), has been approximated to rise to 191.0 million and also 56.3 million, respectively by $2030^{[3]}$.

Diabetic eye disease is a leading cause of vision loss in individuals aged 20 to 74 years [4]. Of the visually disabling problems in individuals with diabetic eye illness, diabetic person macular edema (DME), left neglected, is a common cause of vision loss ${ }^{[3]}$. DME influences central vision as well as can cause decrease in vision ranging from minor visual obscuring to blindness, substantially impacting self-reliance as well as quality of life ${ }^{[5]}$. At least given that the 1980s and until 2010, focal/grid laser photocoagulation was the standard of care to treat macular edema, minimize the danger of vision loss, and also enhance the opportunity of vision gain compared with no therapy ${ }^{[6]}$. More just recently, in phase II and also III tests with ranibizumab as well as aflibercept and phase II trials with bevacizumab, intravitreal shots of anti-vascular endothelial development variable representatives have actually been shown to be superior to focal/grid laser relative to reducing the risk of vision loss as well as raising the possibility of vision gain $^{[7]}$. In intending the demands and also estimating the prices associated with these additional resources, it is important to ascertain a precise estimate of the magnitude of and take the chance of aspects for DME. 
In this review we discuss about the most dangerous risk factors during treatment and in daily life, which can worsen the treatment prognosis. We mention the background of disease and management strategies of risk factors which affect the management.

\section{Methodology:}

PubMed and Embase database were searched up to July, 2018 for relevant studies that discussing the risk factors of glaucoma.

\section{Discussion:}

\section{- Diabetic Macular edema}

DME happens when fluid leakages into the center of the retina at the behind your eye (called the macula), creating it to swell. This swelling obscures your vision and affects your ability to review as well as see information plainly ${ }^{[8]}$. DME happens in both eyes in greater than $50 \%$ of individuals with the condition, although vision could not always be impacted in both ${ }^{[9]}$. If you currently have DME in one eye, speak with your ophthalmologist about just how you could decrease the threat of DME happening in your various other eye.

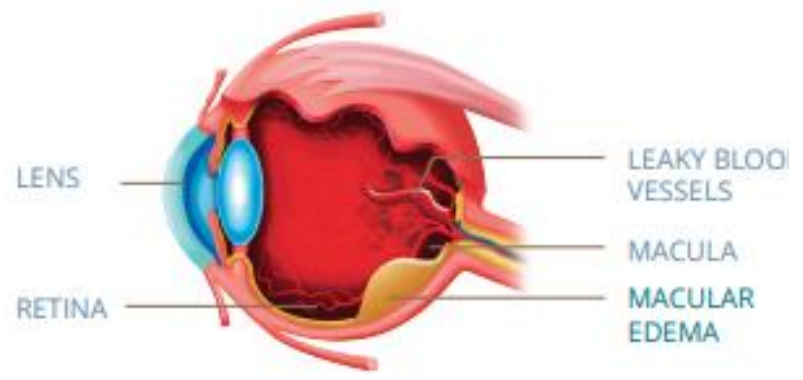

Figure 1. Illustration of Diabetic Macular Edema ${ }^{[8]}$.

\section{- Risk factors and Prevention strategies}

There are a variety of factors both within and also beyond your control that could place you at boosted risk of developing DME, or making it even worse if you currently have it. All patients with kind 1 or type 2 diabetics issues are at threat of creating DME ${ }^{[10]}$.Your degree of danger is related to for how long you have actually had diabetic issues and exactly how well your blood sugar is regulated.

Box1. Risk factors for developing or worsening type 2 diabetes include ${ }^{[10]}$ :

- High cholesterol

- Not getting enough exercise
- Being overweight or obese

- Smoking

Box2. The following are additional risk factors associated with DME ${ }^{[11]}$.

- Diabetic retinopathy severity

- Poor glycemic control

- Age

- Duration of diabetes

- High blood pressure

Box3.Prevention strategies ${ }^{[9]}$ :

- Visit eye doctor regularly - do not miss any scheduled appointments.

- If notice a change in your vision, contact eye doctor immediately.

- Be proactive about vision rehabilitation and the use of visual aids (eg. using a magnifying glass).

- Take your diabetes medications as directed by your doctor.

- Regularly monitor your blood sugar levels. Keeping your levels under control is the single most important way to manage your diabetes.

- Keep high blood pressure under control.

- Maintain a healthy body weight and manage your diet. Your doctor can tell you what you should and shouldn't eat, as well as when you should eat.

- If you smoke, speak with your doctor about a cessation program and work towards quitting as soon as you can.

- Exercise regularly to help avoid high blood pressure and elevated cholesterol.

You will probably be the first person to detect changes in your vision, such as ${ }^{[8]}$ :

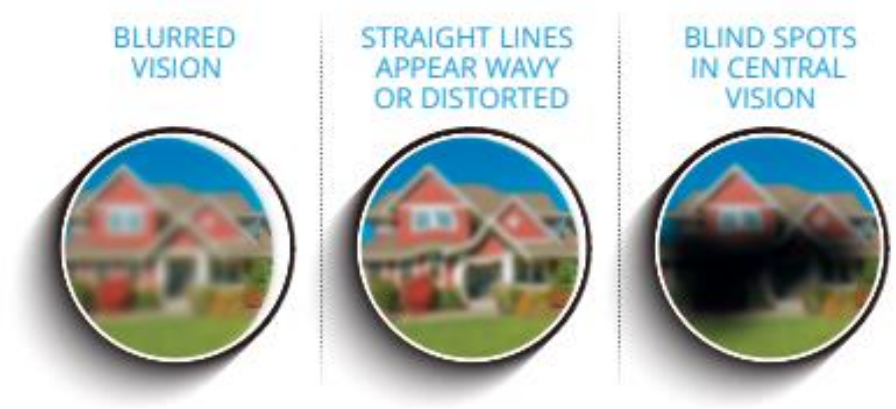

Figure 2.Illustration of changes in vision due to $\mathrm{DME}^{[8]}$. 
Patient have an important role to play in controlling own diabetes, ensuring that vision loss is caught early and getting treated as soon as possible ${ }^{[7]}$.

- Risk factors of DME affecting the treatment prognosis and management of them

\section{Glycemic control}

One of the most well known risk aspect for DME is chronic hyperglycemia generally higher than $120 \mathrm{mg} / \mathrm{dL}$, as well as sometimes over $200 \mathrm{mg} / \mathrm{dL}$ in diabetes. It is believed that continual hyperglycemia directly damages vascular endothelial cells and compromises the blood-retinal barrier (BRB), thereby causing boosted fluid extravasation as well as DME as fluid inflow into the macula exceeds discharge. Particularly, hyperglycemia causes an accumulation of totally free radicals and progressed glycemic end-products (AGE's) and glucotoxins, which form cross-links on proteins, lipids, as well as nucleic acids to change the framework as well as function of these components within the BRB ${ }^{[12]}$. AGE's also cause diabetic microvascular issues by upregulating the expression of vascular endothelial growth variable (VEGF), protein kinase C (PKC), prostaglandins, cytokines, and other vasoactive factors that promote endothelial cell disorder as well as boosted vascular permeability.

Epidemiological research studies have associated period of diabetes mellitus to increased prevalence of DME. The WESDR and also Epidemiology of Diabetes Interventions as well as Complications (EDIC) research study teams reported raised occurrences of DME with longer duration of diabetic issues ${ }^{[13]}$. For instance, in between 4 as well as 14 years of diabetes mellitus duration, the WESDR located that DME enhanced from $8.2 \%$ to $26.1 \%$ of individuals with kind 1 diabetes [21].The EDIC research team discovered that at 4- year follow-up, patients in the initial Diabetes Control and Complications Trial (DCCT) who got intensive insulin therapy (3+ insulin shots and $4+$ glucose measurements daily) showed far better retinopathy outcomes compared to those getting conventional treatment (1-2 insulin shots as well as 1 glucose measurement daily) [13]. Hence, far better management of diabetic issues over time has actually been revealed to lower the risk of DME.

\section{Management:}

One of the most vital markers for glycemic control is glycated hemoglobin (HbAlc), which is agent of ordinary plasma sugar degrees over 3 months. The WESDR located that elevated baseline HbA1c was connected with increased risk of DME ${ }^{[14]}$. Compared with $\mathrm{HbA1c}$ levels $<9.4 \%$, diabetic patients with $\mathrm{HbA} 1 \mathrm{c}$ at $9.5-10.5 \%$ had a 1.90 relative danger of developing DME, while those with $\mathrm{HbA} 1 \mathrm{c}$ procedures of $>12.1 \%$ had a 3.37 relative danger for DME [15]. Likewise, in 2001, a research study of 165 patients by Moreira et al. located that elevated HbAlc was significantly related to DME, while details glucose levels and diabetics issues duration were not [16]. Chou et al. also reported that greater $\mathrm{HbA1c}$ levels correlated with raised DME; in a friend of 102 topics, HbA1c degrees > 8.0\% $(\mathrm{p}=0.005, \mathrm{OR} 4.5)$ were substantially related to raised risk for DME ${ }^{[17]}$. Insulin therapy can be an integral part of glycemic control in both Type 1 as well as Type 2 diabetic issues. However, some studies have revealed that insulin management could actually boost the risk of diabetic retinopathy and DME in particular patients ${ }^{[18]}$. In an animal research by Poulaki et al., acute, intensive insulin therapy in diabetic person rats had the greatest result in triggering BRB breakdown by means of increased VEGF expression [19]. Henricsson et al. located a $100 \%$ enhanced threat of DME (loved one danger 2.0) with insulin therapy compared to oral medicines in 333 insulin-treated patients ${ }^{[20]}$. Likewise, Zapata et al. discovered that, relative to controls, people with Type 2 diabetes mellitus receiving insulin therapy had actually enhanced macular density $(\mathrm{p}=0.036$, OR 1.4) ${ }^{[18]}$. Possible devices of action include raised VEGF expression or vasoreactive impacts of insulin and also abrupt enhancement in glycemic control that further endangers an already-damaged BRB ${ }^{[19]}$. Thus, while insulin administration is necessary in diabetic issues therapy, it is necessary to think about its eye negative effects and potential role in DME.

\section{Hypertension and Hyperlipidemia}

Another threat variable related to raised risk of developing DME is chronic hypertension, which harms endothelial cells, resulting in modifications in the framework of capillary and various other vascular dysfunctions. Numerous research studies have actually 
linked elevated blood pressure with an enhanced risk of DME ${ }^{[21]}$. although it is difficult to develop a cause and effect relationship due to the fact that diabetes as well as hypertension are commonly identical processes. The result of blood pressure control on diabetic retinopathy was reviewed in UKPDS 38, which shows that patients with kind 2 diabetes mellitus assigned to tight blood pressure control had a $37 \%$ decrease in danger of microvascular condition, including retinopathy ${ }^{[22]}$. In a meta-analysis of research studies exploring the impacts of blood pressure control on cardiovascular occasions published in 2016, extensive blood pressure control tended to decrease the danger of progression of retinopathy in individuals with diabetes ${ }^{[23]}$. Nevertheless, a current Cochrane review discloses no significant effects of blood pressure control on progression to proliferative diabetic retinopathy or on scientifically significant macular edema during 4-5 years of follow-up ${ }^{[24]}$.

Hyperglycemia is a recognized risk variable for hyperlipidemia, as triglycerides can be integrated from glucose polymers, thus changing the lipid profile. Although hyperlipidemia is more usual with Type 2 diabetic issues and obesity, Type 1 diabetes can likewise lead to dyslipidemia. Common steps of blood lipid content include overall product cholesterol, lotion low-density lipoprotein (LDL), high-density lipoprotein (HDL), and triglycerides. Hyperlipidemia could be identified by overall cholesterol $>$ 240 (mg/dL), LDL > $160(\mathrm{mg} / \mathrm{dL}), \mathrm{HDL}<40$ $(\mathrm{mg} / \mathrm{dL})$ in males and also $<50(\mathrm{mg} / \mathrm{dL})$ in females, and triglycerides > $200(\mathrm{mg} / \mathrm{dL})$. Chronic dyslipidemia could impact systemic and retinal vasculature in numerous means. Atherosclerotic plaques developed from fatty deposits could occlude vessels, causing hypoxia and also ischemia. Moreover, hyperlipidemia causes endothelial disorder through local inflammatory responses, such as cytokine as well as VEGF upregulation, thus compromising the BRB and resulting in boosted vascular permeability and DME. This would additionally clarify the lipid exudates found in DME after funduscopic retina exam. However, the exact mechanism for the duty of lipids in retinal vascular damage is still unidentified ${ }^{[25]}$.

Management: In the Fenofibrate Intervention and also Event Lowering in Diabetes (FIELD) research, administration of the lipid-lowering representative Fenofibrate considerably reduced the collective threats of retinopathy, proliferative diabetic retinopathy and DME requiring laser treatment in patients with type 2 diabetes, although there were no medically pertinent distinctions in serum lipid parameters in between the Fenofibrate group and also the placebo team at the end of the research study [26]. Nevertheless, the Wisconsin Epidemiologic Study of Diabetic Retinopathy, which assessed the results of lipid control in patients with kind 1 diabetic issues over a duration of 30 years, discloses no significant association between the occurrence of proliferative diabetic retinopathy and/or DME and total cholesterol as well as high-density lipoprotein cholesterol levels, but does reveal an association with $\mathrm{HbAlc}{ }^{[27]}$. In addition, a recent methodical review of the association between dyslipidemia and DME shows that lipid-lowering therapy did not reduce the danger of progression of difficult exudates in DME or the severity of DME ${ }^{[28]}$. Regardless of proof from cohort researches as well as a meta-analysis of situation- control research studies suggesting a strong relationship in between lipid levels and also DME, this was not confirmed by the meta-analysis, which included only potential randomized regulated trials. Yet, when patients with difficult macular exudates were compared with those with regressed macular exudates, product cholesterol, particularly low-density lipoprotein cholesterol, was related to the hard macular exudates ${ }^{[29]}$. Consequently, lipidlowering therapy might need to be considered in the management of DME. High blood pressure and hyperlipidemia are feasible risk factors in the growth and also progression of diabetic retinopathy. Nonetheless, their association with DME is unclear, and better investigation is needed. However, given the well-known cardiovascular problems of diabetes, controls of blood pressure as well as lipids are clearly essential, and optimal management is required.

\section{Nephropathy}

Nephropathy is a well-known microvascular consequence of diabetes that often associates with diabetic retinopathy. Kidney damages is characterized by lowered glomerular filtration rate (GFR), microalbuminuria, as well as feasible end-stage renal illness. A reduced serum protein concentration lowers the plasma 
colloidal osmotic pressure, enhancing edema and liquid retention by Starling's equation. Hence, nephropathy may result in raised BRB vascular damages and also DME as a result of the modification in osmotic balance.

Renal illness is associated with an enhanced likelihood of worsening diabetic retinopathy. In a retrospective research study from the US that entailed greater than 4000 patients with non-proliferative diabetic person retinopathy, the existence of nephropathy boosted the danger of development to proliferative diabetic retinopathy by $29 \%$ (risk ratio 1.29 [95\% selfconfidence period 0.99-1.67]. Although one study reports finding no significant distinctions in systemic factors between ranibizumab-treated patients as well as shamtreated patients, multivariate analysis of the sham-treated patients in the Phase III (RISE/RIDE) studies of ranibizumab shows that a background of renal disease was a significant predictor of poor aesthetic result (best-corrected visual acuity [BCVA] of $20 / 100$ or worse) ${ }^{[30]}$. On top of that, the proportion of patients with serious renal dysfunction (approximated glomerular filtration rate $\backslash 30 \mathrm{~mL} / \mathrm{min} / 1.73 \mathrm{~m} 2$ ) was higher in patients who went through reciprocal vitrectomy than in those that undertook unilateral vitrectomy. In some patients with nephrotic syndrome, therapy of diabetes and nephropathy markedly boosted weight, visual acuity, and also main retinal thickness ${ }^{[30]}$.

\section{Conclusion:}

As its name suggests, diabetic macular edema (DME) is a disease linked to diabetes. Some individuals with diabetes will certainly get a kind of retinopathy-a problem of the retina in the eye. Diabetic retinopathy could progress as well as result in DME (additionally referred to as retinal swelling) that might impact your vision, and is among the most usual reasons for blindness.

Close collaboration in between doctors and also ophthalmologists is important in the managing of patients with diabetic retinopathy and also DME. To make certain very early medical diagnosis as well as treatment all diabetic person patients should be screened for diabetic person retinopathy. Control of blood pressure, serum lipids, and also renal function are very important treatments focused on prevention of visual impairment associated with diabetic retinopathy and DME.
Nevertheless, one of the most vital treatments is the management of blood glucose levels. Intensive follow-up is likewise required in these patients to avoid premature discontinuation of treatment.

It is clear that the variety of people affected by DM is expanding greatly, as is the variety of patients with problems from DM. Fortunately; the eye symptoms of DME can be identified and managed prior to considerable vision loss happens if patients with DM undergo routine eye tests and much better manage their systemic condition.

\section{Reference:}

1. Klein R, Klein B, Moss SE et al. (1984): The Wisconsin epidemiologic study of diabetic retinopathy. Ophthalmology, 91: 1464-1474.

2. Hörle S, Gruner F, Kroll P (2002): Epidemiologie diabetischer Erblindung: eine Übersicht. Klin Monatsbl Augenheilkd, 219: 777784.

3. Hesse L, Grusser M, Hoffstadt $\mathrm{K}$ et al. (2001): Population-based study of diabetic retinopathy in Wolfsburg. Ophthalmology, 98: 1065-1068.

4. Resnikoff S, Pascolini D, Etya'ale D et al. (2004): Global data on visual impairment in the year 2002. Bull World Health Organ., 82(11):844851.

5. Lamoureux EL, Tai ES, Thumboo J et al. (2010): Impact of diabetic retinopathy on vision-specific function. Ophthalmology, 117(4):757765 .

6. Early Treatment Diabetic Retinopathy Study Research Group (1985): Photocoagulation for diabetic macular edema. Early Treatment Diabetic Retinopathy Study report number 1. Arch Ophthalmol., 103(12):1796-1806.

7. Do DV, Nguyen QD, Boyer D et al. (2012): One-year outcomes of the DA VINCI Study of VEGF Trap-Eye in eyes with diabetic macular edema. Ophthalmology, 119(8):16581665.

8. National Eye Institute (2016): Facts about Diabetic Retinopathy. Available at: 
http://www.nei.nih.gov/health/diabetic /retinopathy.asp.

9. Varma R, Bressler NM, Doan QV et al. (2015): Visual Impairment and Blindness Avoided with Ranibizumab in Hispanic and Non-Hispanic Whites with Diabetic Macular Edema in the United States. Ophthalmology, 122(5): 982-989.

10. Royal College of Ophthalmology (2012): Diabetic Retinopathy Guidelines. Available at: https://www.rcophth.ac.uk/wpcontent/uploads/2014/12/2013-SCI301-FINAL-DR-GUIDELINES-DEC2012-updated-July-2013.pdf

11. Eyre H, Kahn R (2004): Preventing cancer, cardiovascular disease, and diabetes. Diabetes Care, 27: 18121824.

12. Romero-Aroca $P$ (2011): Managing diabetic macular edema: the leading cause of diabetes blindness. World J Diabetes, 2(6):98-104

13. The Diabetes Control and Complications Trial/ Epidemiology of Diabetes Interventions and Complications Research Group (2000): Barr CC. Retinopathy and nephropathy in patients with type 1 diabetes four years after a trial of intensive insulin therapy, $\mathrm{N}$ Engl $\mathrm{J}$ Med.,342:381-9.

14. Klein R, Knudtson MD, Lee KE et al. (2009): The Wisconsin Epidemiologic Study of Diabetic Retinopathy XXIII: the twenty-fiveyear incidence of macular edema in persons with type 1 diabetes. Ophthalmology, 116(3):497-503.

15. Klein R, Klein BE, Moss SE et al. (1995): The Wisconsin epidemiologic study of diabetic retinopathy. XV. The longterm incidence of macular edema. Ophthalmology, 102(1):7-16

16. Moreira RO, Trujillo FR, Meirelles RM et al. (2001): Use of optical coherence tomography (OCT) and indirect ophthalmoscopy in the diagnosis of macular edema in diabetic patients. Int Ophthalmol., 24(6):331-6.

17. Chou TH, Wu PC, Kuo JZ et al. (2009): Relationship of diabetic macular oedema with glycosylated haemoglobin. Eye (Lond), 23(6):1360-3.

18. Zapata MA, Badal J, Fonollosa et al. (2010): Insulin resistance and diabetic macular oedema in type 2 diabetes mellitus. $\mathrm{Br} \quad$ J Ophthalmol.,94(9):1230-2.

19. Poulaki V, Qin W, Joussen AM et al. (2002): Acute intensive insulin therapy exacerbates diabetic bloodretinal barrier breakdown via hypoxiainducible factor-1alpha and VEGF. J Clin Invest., 109(6):805-15

20. Henricsson $M$, Nilsson A, Janzon $L$ et al. (1997): The effect of glycaemic control and the introduction of insulin therapy on retinopathy in non-insulindependent diabetes mellitus. Diabet Med.,14(2):123-31.

21. Aroca PR, Salvat M, Fernandez $\mathrm{J}$ et al. (2004): Risk factors for diffuse and focal macular edema. J Diabetes Complications, 18(4):211-5 .

22. UK Prospective Diabetes Study Group (1998): Tight blood pressure control and risk of macrovascular and microvascular complications in type 2 diabetes: UKPDS 38. BMJ., 317:70313.

23. Xie X, Atkins E, Lv J et al. (2016): Effects of intensive blood pressure lowering on cardiovascular and renal outcomes: updated systematic review and meta-analysis. Lancet, 387:43543.

24. Do DV, Wang X, Vedula SS et al. (2015): Blood pressure control for diabetic retinopathy. Cochrane Database Syst Rev., doi: https://doi.org/10.1002/ 14651858.CD006127.pub2.

25. Langeler EG, Snelting-Havinga I, van Hinsbergh VW (1989): Passage of low density lipoproteins through monolayers of human arterial endothelial cells. Effects of vasoactive substances in an in vitro model. Arteriosclerosis, 9(4):550-9.

26. Keech AC, Mitchell P, Summanen PA et al. (2007): Effect of fenofibrate on the need for laser treatment for diabetic retinopathy (FIELD study): a randomised controlled trial. Lancet, 370:1687-97. 
27. Klein BE, Myers CE, Howard KP et al. (2015): Serum lipids and proliferative diabetic retinopathy and macular edema in persons with longterm type 1 diabetes mellitus: the Wisconsin Epidemiologic Study of Diabetic Retinopathy. JAMA Ophthalmol., 133:503-10.

28. Das R, Kerr R, Chakravarthy $\mathrm{U}$ et al. (2015): Dyslipidemia and diabetic macular edema: a systematic review and meta-analysis. Ophthalmology, 122:1820-7.
29. Kameda $Y$, Kumakawa M, Endo N et al. (2010): Association of systemic health and functional outcomes with changes in hard exudates associated with clinically significant macular oedema over the natural course of the disease. Br J Ophthalmol., 94:725-9.

30. Sophie R, Lu N, Campochiaro PA (2015): Predictors of functional and anatomic outcomes in patients with diabetic macular edema treated with ranibizumab.

Ophthalmology, 